\title{
Pocklington's Theorem and Bertrand's Postulate
}

\author{
Marco Riccardi \\ Casella Postale 49 \\ 54038 Montignoso, Italy
}

\begin{abstract}
Summary. The first four sections of this article include some auxiliary theorems related to number and finite sequence of numbers, in particular a primality test, the Pocklington's theorem (see [19]). The last section presents the formalization of Bertrand's postulate closely following the book [1], pp. 7-9.
\end{abstract}

MML identifier: NAT_4, version: 7.7.01 4.66.942

The articles [26], [4], [24], [28], [3], [2], [20], [17], [14], [16], [30], [10], [11], [6], [23], [13], [15], [5], [21], [8], [22], [27], [18], [29], [9], [7], [12], [25], and [31] provide the notation and terminology for this paper.

\section{Some Theorems on Real and Natural Numbers}

The following propositions are true:

(1) For all real numbers $r, s$ such that $0 \leq r$ and $s \cdot s<r \cdot r$ holds $s<r$.

(2) For all real numbers $r, s$ such that $1<r$ and $r \cdot r \leq s$ holds $r<s$.

(3) For all natural numbers $a, n$ such that $a>1$ holds $a^{n}>n$.

(4) For all natural numbers $n, k, m$ such that $k \leq n$ and $m=\left\lfloor\frac{n}{2}\right\rfloor$ holds $\left(\begin{array}{l}n \\ m\end{array}\right) \geq\left(\begin{array}{l}n \\ k\end{array}\right)$.

(5) For all natural numbers $n, m$ such that $m=\left\lfloor\frac{n}{2}\right\rfloor$ and $n \geq 2$ holds $\left(\begin{array}{l}n \\ m\end{array}\right) \geq \frac{2^{n}}{n}$

(6) For every natural number $n$ holds $\left(\begin{array}{c}2 \cdot n \\ n\end{array}\right) \geq \frac{4^{n}}{2 \cdot n}$.

(7) For all natural numbers $n, p$ such that $p>0$ and $n \mid p$ and $n \neq 1$ and $n \neq p$ holds $1<n$ and $n<p$. 
(8) Let $p$ be a natural number. Given a natural number $n$ such that $n \mid p$ and $1<n$ and $n<p$. Then there exists a natural number $n$ such that $n \mid p$ and $1<n$ and $n \cdot n \leq p$.

(9) For all natural numbers $i, j, k, l$ such that $i=j \cdot k+l$ and $l<j$ and $0<l$ holds $j \nmid i$.

(10) For all natural numbers $n, q, b$ such that $\operatorname{gcd}(q, b)=1$ and $q \neq 0$ and $b \neq 0$ holds $\operatorname{gcd}\left(q^{n}, b\right)=1$.

(11) For all natural numbers $a, b, c$ holds $a^{2 \cdot b} \bmod c=\left(a^{b} \bmod c\right) \cdot\left(a^{b} \bmod \right.$ c) $\bmod c$.

(12) Let $p$ be a natural number. Then $p$ is not prime if and only if one of the following conditions is satisfied:

(i) $p \leq 1$, or

(ii) there exists a natural number $n$ such that $n \mid p$ and $1<n$ and $n<p$.

(13) Let $n, k$ be natural numbers. Suppose $n \mid k$ and $1<n$. Then there exists a natural number $p$ such that $p \mid k$ and $p \leq n$ and $p$ is prime.

(14) Let $p$ be a natural number. Then $p$ is prime if and only if the following conditions are satisfied:

(i) $\quad p>1$, and

(ii) for every natural number $n$ such that $1<n$ and $n \cdot n \leq p$ and $n$ is prime holds $n \nmid p$.

(15) For all natural numbers $a, p, k$ such that $a^{k} \bmod p=1$ and $k \geq 1$ and $p$ is prime holds $a$ and $p$ are relative prime.

(16) Let $p$ be a prime number, $a$ be a natural number, and $x$ be a set. Suppose $a \neq 0$ and $x=p^{p \text {-count }(a)}$. Then there exists a natural number $b$ such that $b=x$ and $1 \leq b$ and $b \leq a$.

(17) For all natural numbers $k, q, n, d$ such that $q$ is prime and $d \mid k \cdot q^{n+1}$ and $d \nmid k \cdot q^{n}$ holds $q^{n+1} \mid d$.

(18) For all natural numbers $q_{1}, q, n_{1}$ such that $q_{1} \mid q^{n_{1}}$ and $q$ is prime and $q_{1}$ is prime and $n_{1}>0$ holds $q=q_{1}$.

(19) For every prime number $p$ and for every natural number $n$ such that $n<p$ holds $p \nmid n$ !.

(20) Let $a, b$ be non empty natural numbers. Suppose that for every natural number $p$ such that $p$ is prime holds $p$-count $(a) \leq p$-count $(b)$. Then there exists a natural number $c$ such that $b=a \cdot c$.

(21) Let $a, b$ be non empty natural numbers. Suppose that for every natural number $p$ such that $p$ is prime holds $p$-count $(a)=p$-count $(b)$. Then $a=b$.

(22) For all prime numbers $p_{1}, p_{2}$ and for every non empty natural number $m$ such that $p_{1}{ }^{p_{1}-\operatorname{count}(m)}=p_{2}{ }^{p_{2}-\operatorname{count}(m)}$ and $p_{1}-\operatorname{count}(m)>0$ holds $p_{1}=p_{2}$. 


\section{Pocklington's Theorem}

One can prove the following propositions:

(23) Let $n, k, q, p, n_{1}, p, a$ be natural numbers. Suppose $n-1=k \cdot q^{n_{1}}$ and $k>0$ and $n_{1}>0$ and $q$ is prime and $a^{n-^{\prime} 1} \bmod n=1$ and $p$ is prime and $p \mid n$. Then $p \mid a^{\left(n-^{\prime} 1\right) \div q}-^{\prime} 1$ or $p \bmod q^{n_{1}}=1$.

(24) Let $n, f, c$ be natural numbers. Suppose that

(i) $n-1=f \cdot c$,

(ii) $f>c$,

(iii) $c>0$,

(iv) $\operatorname{gcd}(f, c)=1$, and

(v) for every natural number $q$ such that $q \mid f$ and $q$ is prime there exists a

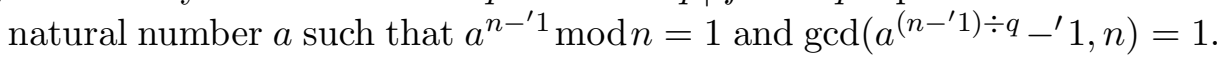
Then $n$ is prime.

(25) Let $n, f, d, n_{1}, a, q$ be natural numbers. Suppose $n-1=q^{n_{1}} \cdot d$ and $q^{n_{1}}>d$ and $d>0$ and $\operatorname{gcd}(q, d)=1$ and $q$ is prime and $a^{n-^{\prime} 1} \bmod n=1$ and $\operatorname{gcd}\left(a^{\left(n-^{\prime} 1\right) \div q}-^{\prime} 1, n\right)=1$. Then $n$ is prime.

\section{Some Prime Numbers}

The following propositions are true:

(26) 7 is prime.

(27) 11 is prime.

(28) 13 is prime.

(29) 19 is prime.

(30) 23 is prime.

(31) 37 is prime.

(32) 43 is prime.

(33) 83 is prime.

(34) 139 is prime.

(35) 163 is prime.

(36) 317 is prime.

(37) 631 is prime.

(38) 1259 is prime.

(39) 2503 is prime.

(40) 4001 is prime. 


\section{Some Theorems on Finite Sequence of Numbers}

One can prove the following propositions:

(41) For all finite sequences $f, f_{0}, f_{1}$ of elements of $\mathbb{R}$ such that $f=f_{0}+f_{1}$ holds $\operatorname{dom} f=\operatorname{dom} f_{0} \cap \operatorname{dom} f_{1}$.

(42) Let $F$ be a finite sequence of elements of $\mathbb{R}$. If for every natural number $k$ such that $k \in \operatorname{dom} F$ holds $F(k)>0$, then $\prod F>0$.

(43) For every set $X_{1}$ and for every finite set $X_{2}$ such that $X_{1} \subseteq X_{2}$ and $X_{2} \subseteq \mathbb{N}$ and $\emptyset \notin X_{2}$ holds $\prod \operatorname{Sgm} X_{1} \leq \prod \operatorname{Sgm} X_{2}$.

(44) Let $a, k$ be natural numbers, $X$ be a set, $F$ be a finite sequence of elements of Prime, and $p$ be a prime number such that $X \subseteq$ Prime and $X \subseteq \operatorname{Seg} k$ and $F=\operatorname{Sgm} X$ and $a=\prod F$. Then

(i) if $p \in \operatorname{rng} F$, then $p$-count $(a)=1$, and

(ii) if $p \notin \operatorname{rng} F$, then $p$-count $(a)=0$.

(45) For every natural number $n$ holds $\prod \operatorname{Sgm}\{p ; p$ ranges over prime numbers: $p \leq n+1\} \leq 4^{n}$.

(46) For every real number $x$ such that $x \geq 2$ holds $\prod \operatorname{Sgm}\{p ; p$ ranges over prime numbers: $p \leq x\} \leq 4^{x-1}$.

(47) Let $n$ be a natural number and $p$ be a prime number. Suppose $n \neq 0$. Then there exists a finite sequence $f$ of elements of $\mathbb{N}$ such that

(i) $\operatorname{len} f=n$,

(ii) for every natural number $k$ such that $k \in \operatorname{dom} f$ holds $f(k)=1$ iff $p^{k} \mid n$ and $f(k)=0$ iff $p^{k} \nmid n$, and

(iii) $p$ - $\operatorname{count}(n)=\sum f$.

(48) Let $n$ be a natural number and $p$ be a prime number. Then there exists a finite sequence $f$ of elements of $\mathbb{N}$ such that len $f=n$ and for every natural number $k$ such that $k \in \operatorname{dom} f$ holds $f(k)=\left\lfloor\frac{n}{p^{k}}\right\rfloor$ and $p$-count $(n !)=\sum f$.

(49) Let $n$ be a natural number and $p$ be a prime number. Then there exists a finite sequence $f$ of elements of $\mathbb{R}$ such that len $f=2 \cdot n$ and for every natural number $k$ such that $k \in \operatorname{dom} f$ holds $f(k)=\left\lfloor\frac{2 \cdot n}{p^{k}}\right\rfloor-2 \cdot\left\lfloor\frac{n}{p^{k}}\right\rfloor$ and $p-\operatorname{count}\left(\left(\begin{array}{c}2 \cdot n \\ n\end{array}\right)\right)=\sum f$.

Let $f$ be a finite sequence of elements of $\mathbb{N}$ and let $p$ be a prime number.

The functor $p$-count $(f)$ yielding a finite sequence of elements of $\mathbb{N}$ is defined by:

(Def. 1) $\operatorname{len}(p$-count $(f))=\operatorname{len} f$ and for every set $i$ such that $i \in$ $\operatorname{dom}(p$-count $(f))$ holds $(p$-count $(f))(i)=p$-count $(f(i))$.

One can prove the following propositions:

(50) For every prime number $p$ and for every finite sequence $f$ of elements of $\mathbb{N}$ such that $f=\emptyset$ holds $p$-count $(f)=\emptyset$.

(51) For every prime number $p$ and for all finite sequences $f_{1}, f_{2}$ of elements of $\mathbb{N}$ holds $p$-count $\left(f_{1} \frown f_{2}\right)=\left(p \text {-count }\left(f_{1}\right)\right)^{\frown}\left(p\right.$-count $\left.\left(f_{2}\right)\right)$. 
(52) For every prime number $p$ and for every non empty natural number $n$ holds $p$-count $(\langle n\rangle)=\langle p$-count $(n)\rangle$.

(53) For every finite sequence $f$ of elements of $\mathbb{N}$ and for every prime number $p$ such that $\prod f \neq 0$ holds $p$-count $\left(\prod f\right)=\sum(p$-count $(f))$.

(54) Let $f_{1}, f_{2}$ be finite sequences of elements of $\mathbb{R}$. Suppose len $f_{1}=\operatorname{len} f_{2}$ and for every natural number $k$ such that $k \in \operatorname{dom} f_{1}$ holds $f_{1}(k) \leq f_{2}(k)$ and $f_{1}(k)>0$. Then $\prod f_{1} \leq \prod f_{2}$.

(55) For every natural number $n$ and for every real number $r$ such that $r>0$ holds $\prod(n \mapsto r)=r^{n}$.

In this article we present several logical schemes. The scheme scheme 1 concerns a ternary predicate $\mathcal{P}$, and states that:

Let $p$ be a prime number, $n$ be a natural number, $m$ be a non empty natural number, and $X$ be a set. If $X=\left\{p^{\prime p^{\prime}-\operatorname{count}(m)} ; p^{\prime}\right.$ ranges over prime numbers: $\left.\mathcal{P}\left[n, m, p^{\prime}\right]\right\}$, then $\prod \operatorname{Sgm} X>0$

for all values of the parameters.

The scheme scheme 2 concerns a ternary predicate $\mathcal{P}$, and states that:

Let $p$ be a prime number, $n$ be a natural number, $m$ be a non empty natural number, and $X$ be a set. If $X=\left\{p^{\prime p^{\prime}-\operatorname{count}(m)} ; p^{\prime}\right.$ ranges over prime numbers: $\left.\mathcal{P}\left[n, m, p^{\prime}\right]\right\}$ and $p^{p-\operatorname{count}(m)} \notin X$, then $p$-count $\left(\prod \operatorname{Sgm} X\right)=0$

for all values of the parameters.

The scheme scheme 3 concerns a ternary predicate $\mathcal{P}$, and states that:

Let $p$ be a prime number, $n$ be a natural number, $m$ be a non empty natural number, and $X$ be a set. If $X=\left\{p^{\prime p^{\prime}-\operatorname{count}(m)} ; p^{\prime}\right.$ ranges over prime numbers: $\left.\mathcal{P}\left[n, m, p^{\prime}\right]\right\}$ and $p^{p \text {-count }(m)} \in X$, then $p$-count $\left(\prod \operatorname{Sgm} X\right)=p-\operatorname{count}(m)$

for all values of the parameters.

The scheme scheme 4 deals with a binary functor $\mathcal{F}$ yielding a set and a binary predicate $\mathcal{P}$, and states that:

Let $n, m$ be natural numbers, $r$ be a real number, and $X$ be a finite set. If $X=\{\mathcal{F}(p, m) ; p$ ranges over prime numbers: $p \leq$ $r \wedge \mathcal{P}[p, m]\}$ and $r \geq 0$, then $\operatorname{card} X \leq\lfloor r\rfloor$

for all values of the parameters.

\section{Bertrand's Postulate}

The following proposition is true

(56) For every natural number $n$ such that $n \geq 1$ there exists a prime number $p$ such that $n<p$ and $p \leq 2 \cdot n$. 


\section{REFERENCES}

[1] M. Aigner and G. M. Ziegler. Proofs from THE BOOK. Springer-Verlag, Berlin Heidelberg New York, 2004.

[2] Grzegorz Bancerek. Cardinal numbers. Formalized Mathematics, 1(2):377-382, 1990.

[3] Grzegorz Bancerek. The fundamental properties of natural numbers. Formalized Mathematics, 1(1):41-46, 1990.

[4] Grzegorz Bancerek. The ordinal numbers. Formalized Mathematics, 1(1):91-96, 1990.

[5] Grzegorz Bancerek. Joining of decorated trees. Formalized Mathematics, 4(1):77-82, 1993.

[6] Grzegorz Bancerek and Krzysztof Hryniewiecki. Segments of natural numbers and finite sequences. Formalized Mathematics, 1(1):107-114, 1990.

[7] Czesław Byliński. Binary operations applied to finite sequences. Formalized Mathematics, 1(4):643-649, 1990.

[8] Czesław Byliński. The complex numbers. Formalized Mathematics, 1(3):507-513, 1990.

[9] Czesław Byliński. Finite sequences and tuples of elements of a non-empty sets. Formalized Mathematics, 1(3):529-536, 1990.

[10] Czesław Byliński. Functions and their basic properties. Formalized Mathematics, 1(1):5565, 1990.

[11] Czesław Byliński. Functions from a set to a set. Formalized Mathematics, 1(1):153-164, 1990.

[12] Czesław Byliński. Some basic properties of sets. Formalized Mathematics, 1(1):47-53, 1990.

[13] Czesław Byliński. The sum and product of finite sequences of real numbers. Formalized Mathematics, 1(4):661-668, 1990.

[14] Agata Darmochwał. Finite sets. Formalized Mathematics, 1(1):165-167, 1990.

[15] Andrzej Kondracki. The Chinese Remainder Theorem. Formalized Mathematics, 6(4):573-577, 1997.

[16] Artur Korniłowicz and Piotr Rudnicki. Fundamental Theorem of Arithmetic. Formalized Mathematics, 12(2):179-186, 2004.

[17] Rafał Kwiatek. Factorial and Newton coefficients. Formalized Mathematics, 1(5):887-890, 1990.

[18] Rafai Kwiatek and Grzegorz Zwara. The divisibility of integers and integer relative primes. Formalized Mathematics, 1(5):829-832, 1990.

[19] W. J. LeVeque. Fundamentals of Number Theory. Dover Publication, New York, 1996.

[20] Takaya Nishiyama and Yasuho Mizuhara. Binary arithmetics. Formalized Mathematics, 4(1):83-86, 1993.

[21] Library Committee of the Association of Mizar Users. Binary operations on numbers. To appear in Formalized Mathematics.

[22] Konrad Raczkowski and Andrzej Nȩdzusiak. Real exponents and logarithms. Formalized Mathematics, 2(2):213-216, 1991.

[23] Piotr Rudnicki and Andrzej Trybulec. Multivariate polynomials with arbitrary number of variables. Formalized Mathematics, 9(1):95-110, 2001.

[24] Andrzej Trybulec. Subsets of complex numbers. To appear in Formalized Mathematics.

[25] Andrzej Trybulec. Binary operations applied to functions. Formalized Mathematics, 1(2):329-334, 1990.

[26] Andrzej Trybulec. Tarski Grothendieck set theory. Formalized Mathematics, 1(1):9-11, 1990.

[27] Andrzej Trybulec. On the sets inhabited by numbers. Formalized Mathematics, 11(4):341$347,2003$.

[28] Michał J. Trybulec. Integers. Formalized Mathematics, 1(3):501-505, 1990.

[29] Wojciech A. Trybulec. Non-contiguous substrings and one-to-one finite sequences. Formalized Mathematics, 1(3):569-573, 1990.

[30] Edmund Woronowicz. Relations and their basic properties. Formalized Mathematics, 1(1):73-83, 1990.

[31] Edmund Woronowicz. Relations defined on sets. Formalized Mathematics, 1(1):181-186, 1990. 